\title{
SÍNDROME DE VOGT-KOYANAGI-HARADA: PRESENTACIÓN DE UN CASO CLÍNICO
}

VOGT-KOYANAGI-HARADA SYNDROME: PRESENTATION OF A CLINICAL CASE

\author{
SÍNDROME DE VOGT-KOYANAGI-HARADA: APRESENTAÇÃO DE UM CASO CLÍNICO
}

\author{
MARÍA CAMILA CHIQUITO FREILE', JUAN PABLO GUTIÉRREZ MENDOZA² \\ ${ }^{1}$ Hospital regional Teodoro Maldonado Carbo, Guayaquil, Ecuador \\ 2 Universidad Católica de Santiago de Guayaquil, Guayaquil, Ecuador
}

Resumen

El síndrome de Vogt-Koyanagi-Harada (VKH) es una entidad clínica caracterizada por panuveítis granulomatosa bilateral difusa. Cursa con desprendimiento exudativo de la retina y puede acompañarse de alteraciones del sistema nervioso central, dermatológicas y auditivas. Aunque es de etiología desconocida, es considerada como una enfermedad autoinmune asociada a los haplotipos HLA-DR4. Se divide en tres fases: prodrómica, oftálmica y de convalecencia. El diagnóstico se realiza mediante la historia clínica y examen físico-oftalmológico completo, así como también por exámenes complementarios como la angiografía retinal fluoresceínica y punción lumbar. El tratamiento se basa en el uso de corticoides tópicos y sistémicos, siendo de gran importancia la dosis inicial y la duración del tratamiento. A continuación se presenta un caso clínico de un paciente de sexo masculino que consulta por disminución de la agudeza visual de ambos ojos y micropsia; mediante criterios clínicos y exámenes complementarios se llegó al diagnóstico de síndrome de Vogt-Koyanagi-Harada en su variante incompleta; él se manejó con corticoterapia tópica y sistémica. Al mismo tiempo este artículo revisa la literatura relacionada con esta patología.

PALABRAS CLAVE: desprendimiento de retina, síndrome uveomeningoencefálico, panuveítis, trastornos de la visión.

Abstract

The Vogt-Koyanagi-Harada syndrome (VKH) is a clinical entity characterized by diffuse bilateral granulomatous pan-uveitis. It is presented with exudative retinal detachment and may be accompanied by dermatologic, auditory and central nervous system alterations. Although it is of unknown etiology, it is considered an autoimmune disease associated with HLA-DR4 haplotypes. It is divided into three phases: prodromal, ophthalmic and convalescence. Diagnosis is performed through clinical history and complete eye examination, as well as by additional tests such as fluorescein angiography and lumbar puncture. The treatment is based on the use of topical and systemic corticosteroids, being the initial dose and duration of treatment of great importance. A clinical case of a male patient who consulted for decreased visual acuity in both eyes and micropsia is presented; by clinical and complementary examinations the diagnosis was Vogt Koyanagi-Harada syndrome in its incomplete form. The patient was treated with topical and systemic corticosteroid therapy. At the same time, this article reviews the literature related to this disease.

KEYWORDS: retinal detachment, uveomeningoencephalitic syndrome, panuveitis, vision disorders.

Resumo

A síndrome de Vogt-Koyanagi-Harada (VKH) é uma entidade clínica caracterizada por panuveíte granulomatosa bilateral difusa. Cursa com 0 desprendimento exsudativo da retina e pode ser acompanhado de alterações do sistema nervoso central, dermatológica e auditiva. Embora seja de etiologia desconhecida, é considerada uma doença autoimune associada com o haplotipo HLA-DRL. Ela é dividida em três fases: prodrômico, oftálmica e convalescença. 0 diagnóstico é feito pela história clínica e exame físico-oftalmológico completo, bem como os exames complementares como a angiografia retiniana fluoresceínica e punção lombar. 0 tratamento é baseado no uso de corticosteroides tópicos e sistêmicos, sendo de grande importância a dose inicial e a duração do tratamento. A seguir é apresentado um caso clínico de um paciente do sexo masculino que consulta pela diminuição da acuidade visual de ambos os olhos e micropsia; utilizando critérios clínicos e exames complementares o diagnóstico foi síndrome de Vogt-Koyanagi-Harada em sua variante incompleta; foi gerido a corticosterapia tópica e sistêmica. Ao mesmo tempo este artigo revisa a literatura relacionada com esta patologia.

PALABRAS-CHAVE: descolamento retiniano, síndrome uveomeningoencefálica, pan-uveíte, transtornos da visão. 
INTRODUCCIÓN

El síndrome de Vogt-Koyanagi-Harada (VKH) es un trastorno multisistémico e idiopático, es de etiología desconocida, aunque se sospecha etiopatología autoinmune, que afecta preferentemente a mujeres jóvenes de piel pigmentada. Se trata de una panuveítis granulomatosa bilateral y difusa que cursa con desprendimiento de retina seroso o exudativo y se acompaña de alteraciones neurológicas, auditivas y cutáneas. ${ }^{1}$

El diagnóstico diferencial del síndrome VKH incluye otras enfermedades que pueden cursar con desprendimiento exudativo de la retina acompañados de panuveítis o uveítis posterior como son: oftalmía simpática, síndromes de puntos blancos evanescentes, coroidopatía geográfica, escleritis posterior, linfoma primario intraocular de células $\mathrm{B}$, epiteliopatía pigmentaria placoidea multifocal aguda, entre otras. ${ }^{2}$ Existe una asociación con el haplotipo HLA-DR4, incluso se ha definido un nuevo autoantígeno KU-MEL-1 que forma parte del HLADRB1 que pudiera tener gran importancia en la patogenia de la enfermedad. .,4 $^{3}$

A continuación se presenta un caso clínico de un paciente masculino de 25 años de edad, quien consulta a la emergencia del servicio de oftalmología por presentar disminución de la agudeza visual bilateral acompañado de manifestaciones oculares, sistémicas y signos de meningismo. Lo interesante de este caso es que el paciente acudió al hospital a los dos días de iniciado los síntomas, lo cual nos permitió llegar a un diagnóstico precoz y tratamiento oportuno con corticoterapia, evitando así las complicaciones tanto sistémicas como oftalmológicas que en ciertos casos puede ocurrir en los individuos afectados.

\section{EXPOSICIÓN DEL CASO CLÍNICO}

Paciente de sexo masculino de 25 años de edad con antecedente patológico de linfoma de Hodgkin diagnosticado y tratado en la infancia. Consulta al servicio de oftalmología del hospital Teodoro Maldonado Carbo por presentar disminución de la agudeza visual bilateral de dos días de evolución, acompañado de micropsia, precedido de malestar general, cefalea holocraneana de alta intensidad, alza térmica no cuantificada e irritabilidad.

Al examen físico la presión arterial 110/80 $\mathrm{mmHg}$, temperatura $37.9 \mathrm{C}^{\circ}$, frecuencia cardiaca y respiratoria dentro de límites normales. Al examen físico general se observa al paciente irritable, Glasgow 15/15, sin alteraciones dermatológicas ni auditivas. Los exámenes de laboratorio como biometría hemática completa, parámetros bioquímicos y examen elemental de orina se encontraron dentro de rangos normales. Al mismo tiempo no se encontró cambios llamativos en la resonancia magnética de cerebro, ni en la radiografía estándar de tórax.

A la exploración oftalmológica el paciente presenta agudeza visual (medido con cartilla de Snellen) de su ojo derecho 20/30 y ojo izquierdo 20/100; la presión intraocular de ambos ojos fue $15 \mathrm{mmHg}$. En la biomicroscopía con lámpara de hendidura no se observaron alteraciones en el segmento anterior de los ojos. Al fondo ocular se evidenciaron áreas blanquecinas exudativas maculares y paramaculares con líquido subretinal, de predominio izquierdo (figura 1).
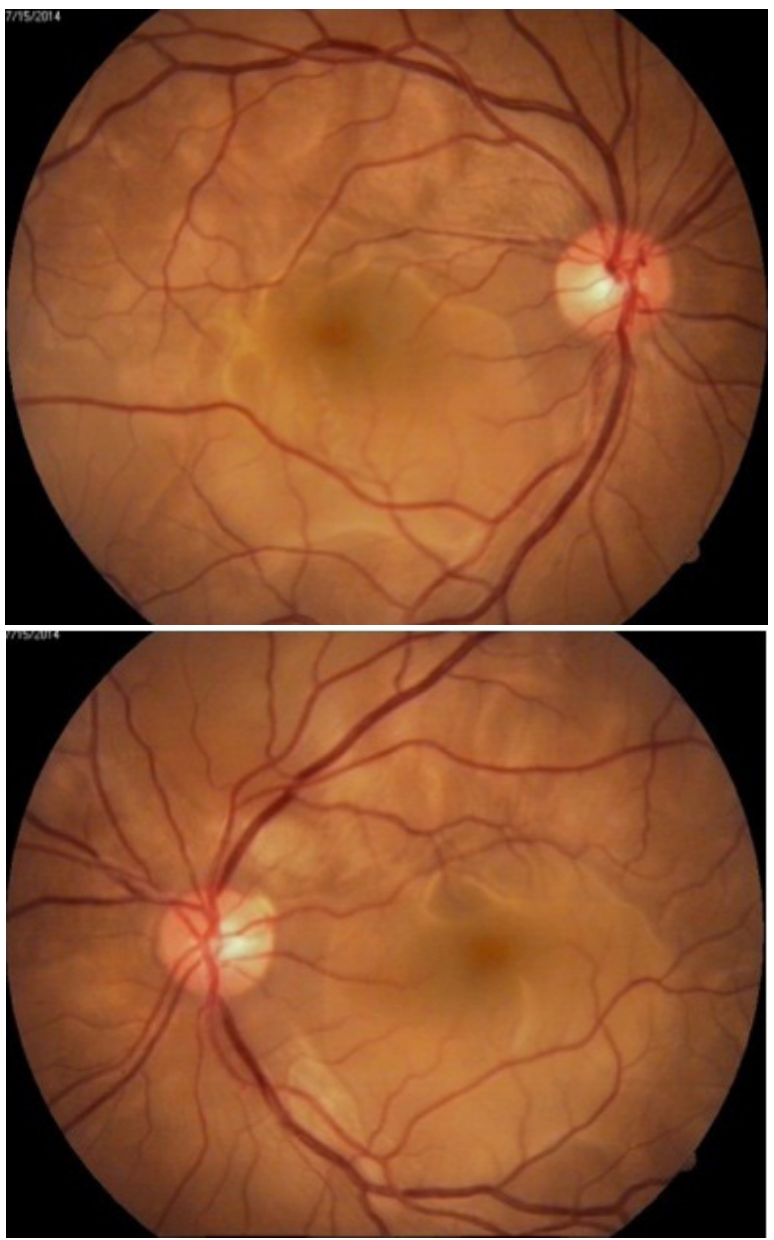

Figura 1. Retinografía de ojo derecho e izquierdo donde se observa desprendimiento exudativo de la retina.

Fuente: servicio de Oftalmología hospital Teodoro Maldonado Carbo. 
En la angiografía retinal fluoresceínica se observa separación de las capas de la retina exudativa de ambos ojos y múltiples áreas de fugas puntuales de hiperfluorescencia a la altura del epitelio pigmentario de la retina (figura 2).
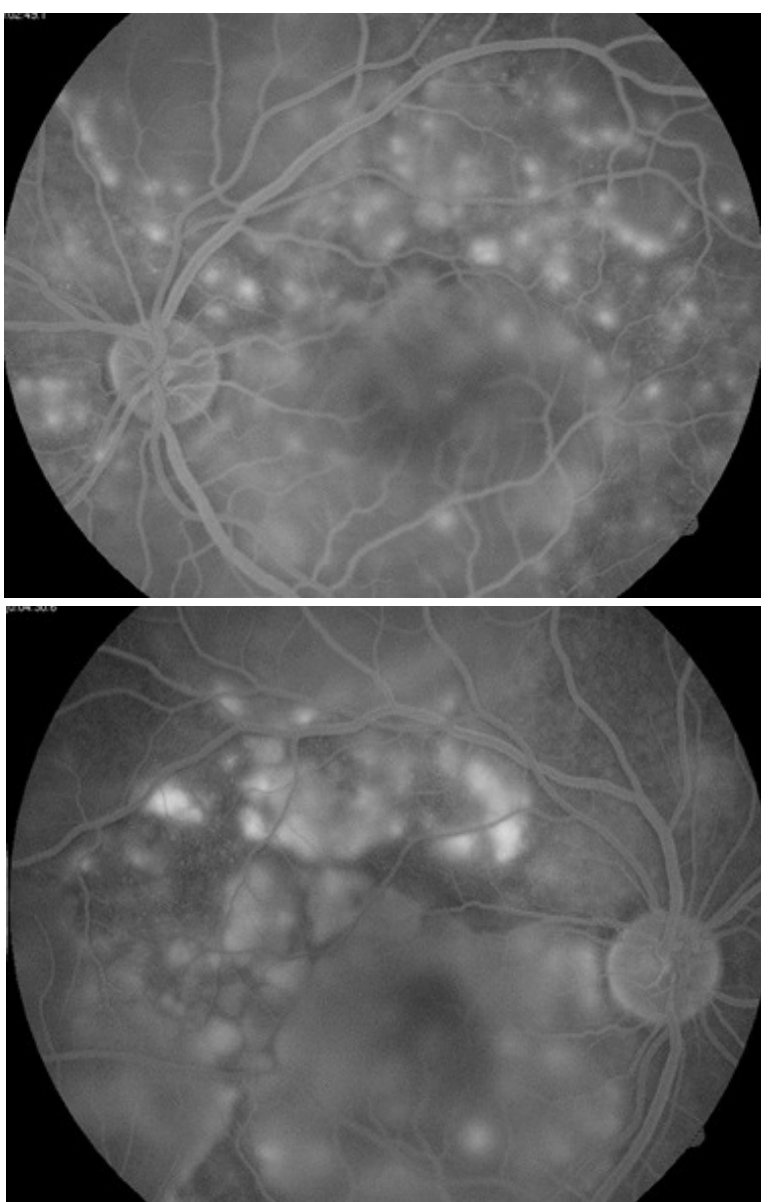

Figura 2. Angiografía retinal fluorosceínica de ojo derecho e izquierdo donde se observan múltiples áreas hiperfluorescentes de fuga en el polo posterior. Fuente: servicio de Oftalmología hospital Teodoro Maldonado Carbo.

En la ecografía ocular hubo engrosamiento coroideo posterior junto con desprendimiento de la retina con líquido subyacente bilateral (figura 3). Se interconsultó al servicio de Neurología para efectuar una punción lumbar, la cual reveló: proteinorraquia de $230 \mathrm{mg} / \mathrm{dl}$ y leucocitos 5/campo.

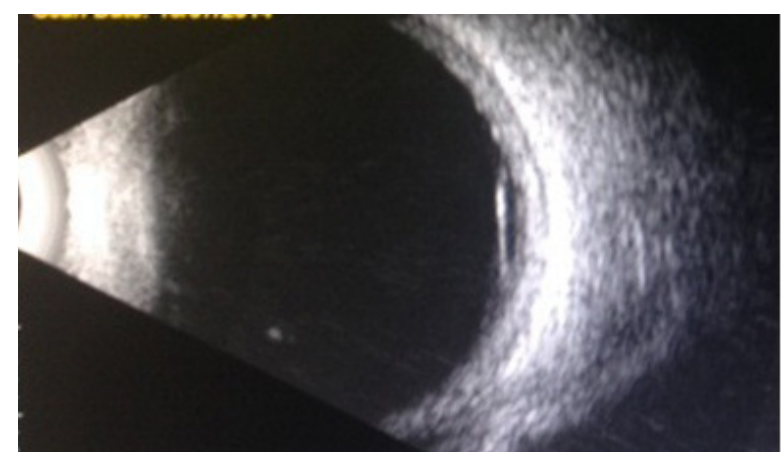

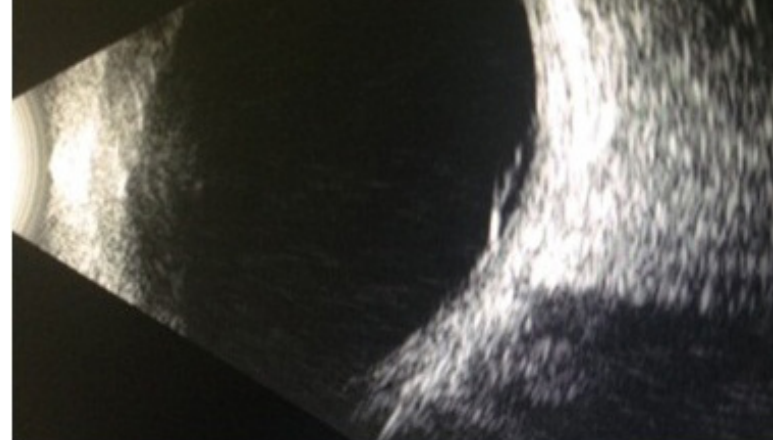

Figura 3. Ecografía ocular de ojo derecho e izquierdo donde se observa separación de las capas de la retina con líquido subyacente.

Fuente: servicio de Oftalmología hospital Teodoro Maldonado Carbo.

\section{DISCUSIÓN}

Con el caso clínico presentado se recalca el beneficio del diagnóstico precoz en este tipo de patología, con la consecuente instauración del tratamiento oportuno evitando así las complicaciones, como la afectación en cámara anterior.

El diagnóstico es principalmente clínico, siendo necesario realizar el diagnóstico diferencial con oftalmia simpática, coriorretinopatía serosa central, tumores coroideos, síndrome de efusión uveal, epiteliopatía pigmentaria placoidea multifocal aguda, entre otros. ${ }^{5-7}$ Existen cinco criterios clínicos que ayudan a establecer las tres formas clínicas del síndrome VKH y permiten descartar los otros diagnósticos diferenciales, en dependencia de la presencia o ausencia de estos criterios (tabla).

\section{TABLA. CRITERIOS DIAGNÓSTICO PARA EL SÍNDROME DE VKH}

1. No antecedentes personales de trauma o cirugía ocular previos al cuadro uveal

2. No evidencias clínicas o de laboratorio de otras causas de uveítis

3. Condición ocular bilateral la cual puede presentarse como manifestaciones oculares precoces 0 tardías.

4. Hallazgos neurológicos 0 auditivos manifiestos en etapas iniciales 0 bien documentados como: meningismo, tinitus o pleocitosis en LCR.

5. Hallazgos cutáneos, que no anteceden al inicio de las alteraciones neurológicas, como alopecia, poliosis o vit́lígo simétrico.

Por lo tanto se considera VKH completo cuando los cinco criterios están presentes; VKH incompleto, cuando los criterios del 1 al 3 se encuentran presentes obligatoriamente y del 4 al 5 pueden estar o no presentes; y por último VKH probable cuando se trata de una condición ocular aislada teniendo presentes solo los criterios del 1 al 3. ${ }^{9}$ En el caso descrito en este artículo, el paciente no tenía antecedentes de trauma o cirugía ocular y tampoco hubo evidencias clí- 
nicas o de laboratorio de otros tipos de uveítis; sin embargo, el paciente presentó manifestaciones oculares precoces comprobadas por angiografía retinal fluoresceínica y ecografía modo B, además de signos de meningismo; siendo por esto clasificado como síndrome de VKH incompleto.

Por otra parte, esta entidad clínica se divide en tres fases dependiendo del tiempo de evolución de la enfermedad: la prodrómica, oftálmica y de convalecencia. En la fase prodrómica los pacientes pueden presentar cefalea, naúseas, vértigo, fiebre y pleocitosis en LCR; dura de 1 a 4 semanas. Luego comienza la fase oftálmica con visión borrosa, disminución de la agudeza visual bilateral y hallazgos oculares, principalmente desprendimiento seroso de la retina bilateral, uveítis bilateral y puede haber un aumento de la presión intraocular; esta fase puede durar de 2 a 3 meses. Y por último la fase de convalecencia que suele aparecer a los 3 meses de la fase aguda y consiste principalmente en síntomas cutáneos, como canicie, poliosis, alopecía y vitíligo. ${ }^{10}$

El tratamiento indicado en el síndrome de VKH son los corticoesteroides tópicos como el acetato de metilprednisolona al $1 \%$ indicado en colirio a dosis de una gota cada hora en ambos ojos, así como también el uso de corticoesteroides sistémicos de 60 a $100 \mathrm{mg}$ (ajustado al peso del paciente a $1 \mathrm{mg} / \mathrm{kg}$ ) vía oral como dosis inicial y luego ir disminuyendo muy lentamente la dosis para evitar los efectos no deseados de la corticoterapia.

Es primordial individualizar la dosis inicial, duración y velocidad de retirada de los corticoides según lo necesita cada paciente basándose en la mejoría visual y la mejoría tanto de las manifestaciones oculares como sistémicas. ${ }^{11}$ Hay que considerar el uso de inmunosupresores en pacientes que no pueden tolerar los corticoesteroides sistémicos o que no respondan a ellos.

Por último, es fundamental el uso de ciclopléjicos tópicos como tropicamida o atropina en casos de afectación importante de la cámara anterior o segmento anterior. ${ }^{11,12}$ El uso de corticoides sistémicos e inmunosupresores ha mejorado sustancialmente el pronóstico visual de los pacientes con VKH cuando el diagnóstico es oportuno y precoz.
Las complicaciones se correlacionan con la duración de la enfermedad, el número de recurrencias y la edad del paciente en el momento del inicio de la enfermedad. Estas incluyen catarata, glaucoma, neovascularización coroidea y fibrosis retiniana, así como atrofia óptica y alteraciones pigmentarias de la retina. A pesar de que dichas complicaciones pueden ser manejadas mediante tratamientos médicos y quirúrgicos, el pronóstico visual de dichos pacientes puede verse afectado si no se tratan de manera oportuna. ${ }^{13}$ Existen 3 factores predictivos de mal pronóstico visual según Rubsamen y Gass: la edad avanzada al inicio de la enfermedad, inflamación crónica que requiera tratamiento prolongado con corticoides y la presencia de neovascularización coroidea. ${ }^{14,15}$

\section{CONCLUSIONES}

En el caso presentado se permitió instaurar el tratamiento de forma rápida y evitar el desarrollo de diferentes complicaciones. Se debe vigilar a los pacientes hasta dos años después de la fase aguda ya que puede existir en ciertos casos una fase crónica recurrente que puede ser resistente al tratamiento esteroideo. Existen criterios diagnósticos del VKH, y lo más importante es recordar que hay síntomas sistémicos, no solo oculares. Sin embargo, en las etapas iniciales las manifestaciones clínicas no son tan floridas y es de mayor relevancia la historia clínica junto con la valoración oftalmológica completa. El pronóstico visual de los pacientes es generalmente bueno si el diagnóstico es temprano y se prescribe un tratamiento con corticoesteroides sistémicos y tópicos adecuado de forma agresiva y mantenida en el tiempo, y recordar que los inmunomodulares son una opción terapéutica. ${ }^{1}$

\section{REFERENCIAS BIBLIOGRÁFICAS}

1. De Domingo B, Blanco M, Rodríguez C, Piñeiro A. Síndrome de Vogt Koyanagi Harada. Arch Soc Esp Oftalmol. 2008;83:385-90.

2. Martínez A, Eguiluz S, Artaraz J, Fonolfosa A. Enfermedad de Vogt-Koyanagi-Harada: descripción de diez casos. Rev Soc Med Interna Aragón Navar Rioja País Vasco. 2007;17-24.

3. Riveros Frutos A, Romera Romero P, Holgado Pérez S, Anglada Escalona JR, Martínez-Morillo M, Tejera Segura B. Enfermedad de Vogt-Koyanagi-Harada. Semin Fund Esp Reumatol. octubre de 2012;13(4):142-6.

4. Ramírez-Rosales A, Góngora-Rivera F, García-Pompemayer M, Rodríguez-Robles L, Velarde-Magaña G. Enfermedad 
de Vogt-Koyanagi-Harada: Reporte de un caso. Rev Mex Neurocienc. 2012;13(5):275-80.

5. Zanolli M, Oporto C, Castiglione C. Enfermedad de Vogt Koyanagi Harada en una serie clínica de pacientes Red de Salud UC: Clasificación y Evolución Clínica. Rev Anacem. 2007;1:40-3.

6. Ng TP, Feng L, Yap KB, Lee TS, Tan CH, Winblad B. Long-term metformin usage and cognitive function among older adults with diabetes. J Alzheimers Dis JAD. 2014;41(1):61-8.

7. Pérez Padilla C, Oñoz Gálves Y, Padierne Gonzáles N, Herrera Lazo Z, Pita Alemán N, Pérez Pérez R. Síndrome de Vogt-Koyanagi-Harada probable. Presentación de un caso pediátrico. Mediciego. 2013;19(2).

8. Andreoli CM, Foster CS. Vogt-Koyanagi-Harada disease. Int Ophthalmol Clin. 2006;46(2):111-22.

9. Triana-Casado I, Molina-Cisneros C. Probable síndrome de Vogt-Koyanagi-Harada; informe de un caso. Rev Mex Oftalmol. el 1 de julio de 2011;85(03):156-60.

10. Castillo Castañeda AM, González Coraspe JA, Parada Sánchez E. Síndrome de Vogt Koyanagi Harada Incompleto: A propósito de un caso. CIMEL Cienc E Investig Médica Estud Latinoam [Internet]. 2010 [citado el 21 de diciembre de 2015];15(2). Recuperado a partir de: http://www.redalyc.org/articulo.oa?id=71721155011
11. Rubsamen PE, Gass JD. Vogt-Koyanagi-Harada syndrome. Clinical course, therapy, and long-term visual outcome. Arch Ophthalmol Chic Ill 1960. mayo de 1991;109(5):682-7.

12. Coto H, Rao NA. Sympathetic ophthalmia and VogtKoyanagi-Harada syndrome. Int Ophthalmol Clin. 1990;30(4):279-85.

13. Read RW, Holland GN, Rao NA, Tabbara KF, Ohno S, Arellanes-Garcia L, et al. Revised diagnostic criteria for Vogt-Koyanagi-Harada disease: report of an international committee on nomenclature. Am J Ophthalmol. mayo de 2001;131(5):647-52.

14. Read RW, Rechodouni A, Butani N, Johnston R, LaBree LD, Smith RE, et al. Complications and prognostic factors in Vogt-Koyanagi-Harada disease. Am J Ophthalmol. mayo de 2001;131(5):599-606.

15. Al-Kharashi AS, Aldibhi H, Al-Fraykh H, Kangave D, Abu El-Asrar AM. Prognostic factors in Vogt-Koyanagi-Harada disease. Int Ophthalmol. junio de 2007;27(2-3):201-10 